\title{
SÍNDROME DE MIRIZZI: REPORTE DE UN CASO
}

\author{
MIRIZZI SYNDROME: A CASE REPORT
}

\section{Montenegro, Rosangel*; Ojeda Mariana*; Parekh, Nabil*; Morales, Danilo*}

*Estudiante del X semestre de Medicina, Universidad de Panamá, Ciudad de Panamá

Recibido: 26 de diciembre del 2019

Aceptado: 17 de mayo del 2020

Montenegro R, Ojeda M, Parekh N, Morales D. Síndrome de Mirizzi: Reporte de un Caso. Rev Méd Cient. 2020; 33(2):26-40. DOI:

$10.37416 /$ rmc.v33i2.541

\section{RESUMEN}

Se presenta el caso de una paciente femenina de 38 años de edad bajo observación del Servicio de Gastroenterología desde inicios de septiembre del 2019, por dilatación intra y extrahepática debido a un cálculo en el tercio medio del colédoco y la vesícula sobredistendida con cuatro cálculos en su interior. Se realizó colangiopancreatografía retrógrada endoscópica (CPRE) en dos ocasiones, con la sospecha de síndrome de Mirizzi tipo II; una colecistectomía parcial con extracción de varios cálculos, uno de ellos enclavado en el conducto cístico que condicionaba a una comunicación con la vía biliar; y una colangiografía intraoperatoria que documentó un cálculo en la vía biliar distal, por lo cual se realizó exploración abierta y se extrajo dicho cálculo. Posteriormente, se le dejó tubo de Kher en la vía biliar y se realizó una colangiografía final a través del mismo. El síndrome de Mirizzi fue descrito por primera vez en el año de 1947 por Pablo Mirizzi y señala factores etiológicos de colestasis extrahepática en algunos pacientes con litiasis vesicular. ${ }^{1}$ Esta patología es infrecuente a nivel mundial.

Palabras claves: Síndrome de Mirizzi, ictericia, colédoco

\section{ABSTRACT}

We present the case of a 38-year-old female patient under observation by the Gastroenterology service since the beginning of September 2019, which reveals intra and extrahepatic bile duct dilation conditioned by gallstones in the middle third of the common bile duct, as well as an overdistended gallbladder with four calculi inside. Endoscopic retrograde cholangiopancreatography (ERCP) was performed on two occasions, with suspicion of Mirizzi syndrome type II, partial cholecystectomy was performed with the extraction of several stones, one of them was nailed in the cystic duct conditioning communication with the bile duct, an intraoperative cholangiography results in a stone in the distal bile duct, which is why the open exploration was performed and the stone was extracted; later, a Kher tube was left in the bile duct and a final cholangiography was performed through it. Mirizzi syndrome was first described by Pablo Mirizzi in 1947 and the etiological factors of extrahepatic cholestasis in some patients with gallbladder lithiasis. 1 This pathology is rare worldwide.

Key words: Mirizzi syndrome, jaundice, common bile duct.

SÍNDROME DE MIRIZZI: REPORTE DE UN CASO by Montenegro R, Ojeda M, Parekh N, Morales D, is licensed under a Creative Commons Attribution-NonCommercial-NoDerivs 4.0 Unported License. Permissions beyond the scope of this license may be available at www.revistamedicocientifica.org. 


\section{INTRODUCCIÓN}

La enfermedad por cálculos es una de las causas principales de intervención quirúrgica del árbol biliar, siendo la forma de presentación más común la colecistitis aguda; sin embargo, pueden presentarse manifestaciones poco usuales como el síndrome de Mirizzi. ${ }^{2}$

La descripción de la obstrucción parcial de la vía biliar secundaria a un cálculo impactado e inflamación fue inicialmente realizada por Hans Kehr en 1905, Ruge en 1908, Mirizzi detalla por primera vez esta patología en 1940, Levrot en 1941, y en 1948 Mirizzi realizó una publicación de este síndrome, que lo hace famoso y se adjudica el epónimo de síndrome de Mirizzi a esta entidad. ${ }^{1}$

Se define hoy día como la compresión extrínseca de la vía biliar secundaria a la impactación de un cálculo ya sea en la bolsa de Hartmann, en el infundíbulo de la vesícula o en el conducto cístico. Se presenta usualmente en mujeres, con una frecuencia menor al $1 \%$. La manifestación clínica más común es la ictericia obstructiva, asociada a dolor en el hipocondrio derecho, y en ocasiones puede ser tan grave que puede manifestarse como un cuadro de colangitis. Además, a lo largo de la historia se han hecho varias clasificaciones del mismo. En la actualidad la más utilizada es la Csendes, la cual es validada en 2008 por Csendes y Beltrán, donde se encuentran cinco tipos diferentes dependiendo de la presencia $o$ no de fístula colecistobiliar o colecistoentérica. ${ }^{3,4}$

Se requiere alta sospecha del mismo para su diagnóstico. Son útiles como herramientas imagenológicas el ultrasonido hepatobiliar (USG), la colangiopancreatografía retrógrada endoscópica (CPRE), colangioresonancia (CRM) o colangiografía directa.
La tomografía es de ayuda sobre todo para diferenciar un compromiso neoplásico de la vía biliar. El tratamiento quirúrgico es el de elección. ${ }^{5}$

Imagen 1. Pablo Luis Mirizzi (1893-1964).

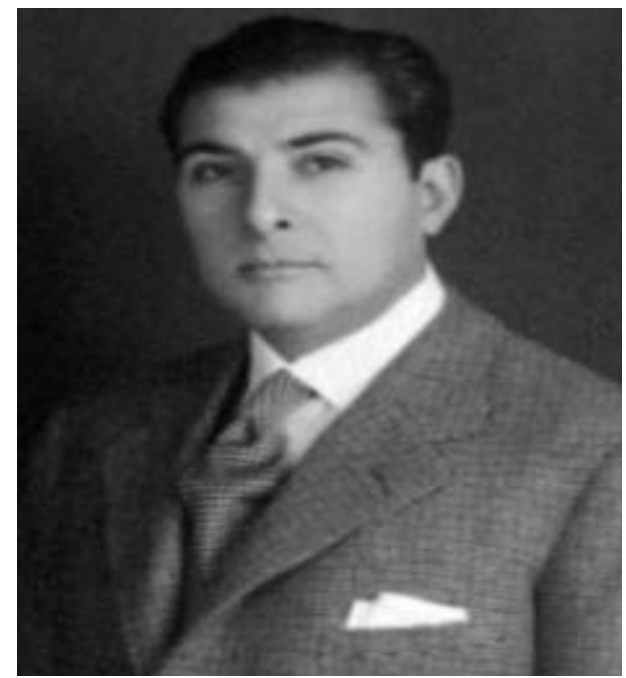

Fuente: Tomado de Cervantes J. Coledocolitiasis: Evolución del diagnóstico y tratamiento. Cir Gen 2003; 25:35-40

\section{CASO CLÍNICO}

\section{ENFERMEDAD ACTUAL}

Paciente femenina de 38 años de edad sin antecedentes personales patológicos, con historia de 1 mes de evolución de dolor tipo cólico, en hipocondrio derecho con irradiación a región lumbar, de intensidad leve a moderada, no asociado a la ingesta, sin exacerbantes, ni atenuantes; acompañado de náuseas y vómitos de tipo bilioso durante 3 días, por lo que consultó en múltiples ocasiones en la policlínica donde es tratada como gastritis, sin mejoría.

La paciente refirió aumento de la intensidad del dolor a 9/10, con persistencia de náuseas, vómitos y ahora acompañado de ictericia de piel y escleras por lo que es referida a cuarto de urgencias del hospital. Posteriormente, se le realizó USG hepatobiliar que reportó colelitiasis y dilatación de la vía biliar intra y extrahepática, siendo admitida por el servicio de gastroenterología por sospecha de coledocolitiasis. 


\section{HISTORIA ANTERIOR}

Niega consumo de tabaco, alcohol o drogas. Niega antecedentes patológicos personales y familiares.

\section{INTERROGATORIO POR APARATOS Y SISTEMAS}

Paciente con ictericia y dolor abdominal al colocarse en posición decúbito lateral derecho. Resto del interrogatorio sin datos de relevancia.

\section{EXAMEN FÍSICO}

Se encuentra alerta, orientada y consciente.

PA: 123/76 T: 36.5ㅇ FC: 82 lpm FR: 18 rpm

Al examen físico se encuentran escleras ictéricas; y abdomen blando, depresible con ruidos hidroaéreos presentes, doloroso a la palpación profunda en hipocondrio derecho, no difuso, sin rebote, no se palpan masas ni visceromegalias. Resto sin datos de relevancia.

\section{ESTUDIOS DE LABORATORIO}

Al ingreso se realiza hemograma completo, sin alteraciones. Los resultados de la química sanguínea se pueden ver en la Tabla 1.

\section{ESTUDIOS DE IMAGEN}

Se le realiza USG que revela dilatación de las vías biliares intra y extrahepática condicionada por cálculo en el tercio medio del colédoco y vesícula sobredistendida con cuatro cálculos en su interior (ver imagen 2) de $<1.4 \mathrm{~cm}$ que se movilizan con los cambios posturales. Se lleva el 11 de septiembre de 2019 a realizar CPRE (ver imagen 3) donde se reporta papila de Vater con ectropión y vía biliar intra y extrahepática dilatada. Esta última con gran defecto de llenado, $8 \mathrm{~mm}$ en colédoco medio y con un diagnóstico de coledocolitiasis no resuelta, por lo que se programa otra CPRE para el 13 de septiembre en la cual se reporta papila de Vater con prótesis en su interior y vía biliar intrahepática de $5 \mathrm{~mm}$ sin defecto de llenado. Se excluye el conducto cístico por probable lito en su interior y se deja en observación por síndrome de Mirizzi.
Tabla 1. Química Sanguínea al Ingreso

\begin{tabular}{ll}
\hline \multicolumn{2}{c}{ Química Sanguínea } \\
\hline Glucosa & $106.2 \mathrm{mg} / \mathrm{dL}$ \\
Bilirrubina directa & $7.5 \mathrm{mg} / \mathrm{dL}$ \\
Bilirrubina indirecta & $1.4 \mathrm{mg} / \mathrm{dL}$ \\
Bilirrubina total & $8.9 \mathrm{mg} / \mathrm{dL}$ \\
Deshidrogenasa Láctica & $321 \mathrm{U} / \mathrm{L}$ \\
OXAL-ACET & - \\
GLUT/PIRUV & $653 \mathrm{U} / \mathrm{L}$ \\
Amilasa & $63 \mathrm{U} / \mathrm{L}$ \\
\hline
\end{tabular}

Fuente: Expediente Servicio de Cirugía.

Complejo Hospitalario Dr. Arnulfo Arias Madrid.

Imagen 2. Litos en vesícula biliar

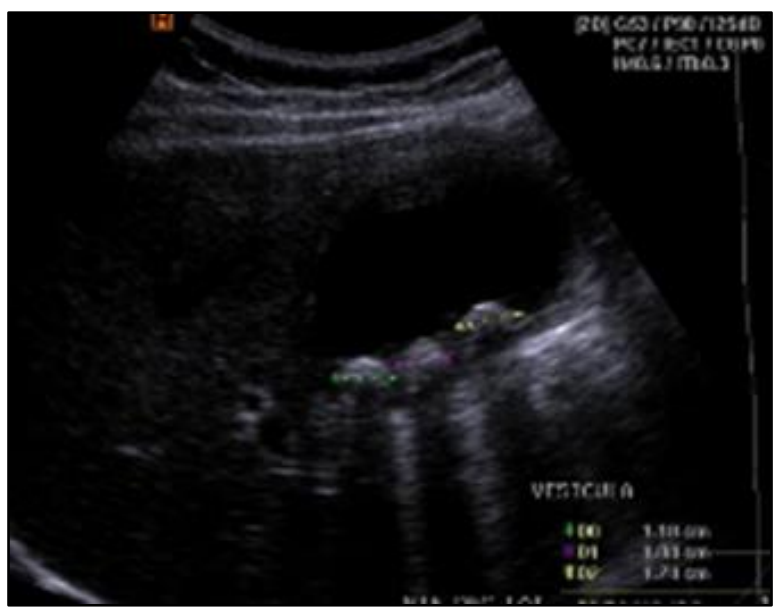

Fuente: Imagen proporcionada por el Servicio de Cirugía del Complejo Hospitalario Dr. Arnulfo Arias Madrid.

Imagen 3. CPRE

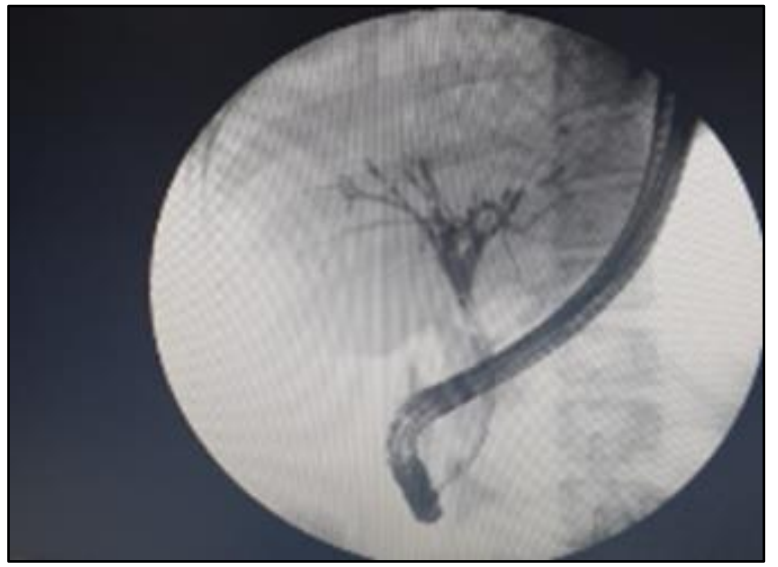

Fuente: Imagen proporcionada por el Servicio de Cirugía del Complejo Hospitalario Dr. Arnulfo Arias Madrid. 


\section{TRATAMIENTO}

Paciente recibe ciprofloxacina $800 \mathrm{mg}$ IV, metronidazol $1500 \mathrm{mg} \mathrm{IV}$, ranitidina $100 \mathrm{mg}$ IV y acetaminofén a demanda por dolor. Se le realizó colecistectomía parcial, extracción de cálculos, exploración de vías biliares abierta, colocación de tubo de Kehr y colangiografía posterior. Se extraen seis litos de la vesícula, y un lito enclavado en conducto cístico sin observar plano de separación con la vía biliar. Al realizar colangiografía se evidenció defecto de llenado en colédoco distal, por lo cual se realizó exploración de vía biliar abierta más extracción de cálculo, coledocorrafia y colocación de tubo de Kehr. A través de este último, se realiza colangiografía final evidenciando adecuado paso de contraste hacia el duodeno sin defectos de llenado en la vía biliar. Se deja drenaje de Jackson Pratt.

\section{DIAGNÓSTICO DIFERENCIAL}

- Colecistitis aguda litiásica.

- Coledocolitiasis.

- Síndrome de Mirizzi.

La coledocolitiasis se refiere a la presencia de cálculos biliares en el conducto biliar común, la cual se corrobora realizando ultrasonido transabdominal del cuadrante superior derecho donde se evidencia conducto biliar común dilatado, dilatación biliar intrahepática y piedra oclusiva del conducto biliar común, ${ }^{6}$ que a diferencia del síndrome de Mirizzi, no se observa.

La colecistitis aguda litiásica es la inflamación de la vesícula biliar por la obstrucción de un cálculo biliar. El abordaje inicial incluye ecografía del cuadrante superior derecho, que mostraría distensión de la vesícula biliar, engrosamiento de la pared vesicular, edema y líquido pericolequístico. ${ }^{7}$
El síndrome de Mirizzi presenta clínica parecida a la coledocolitiasis, pero en estudios imagenológicos, utilizando colangiopancreatografía retrógrada endoscópica, se evidencia estrechamiento del conducto hepático común, cálculos en el conducto cístico y dilatación del árbol biliar intrahepático. ${ }^{3,8}$

Imagen 4: Vesícula biliar y cálculos

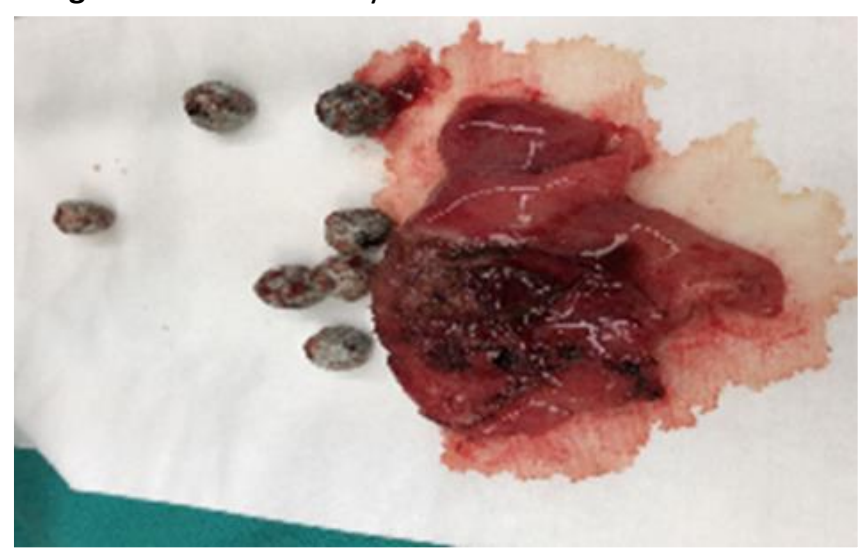

Fuente: Imagen proporcionada por el Servicio de Cirugía del Complejo Hospitalario Dr. Arnulfo Arias Madrid.

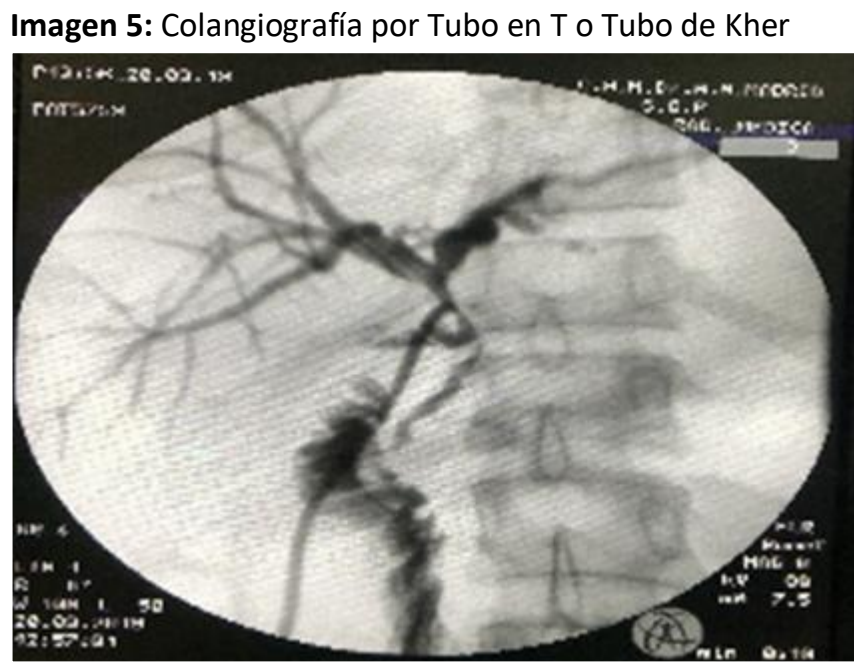

Fuente: Imagen proporcionada por el servicio de Cirugía del Complejo Hospitalario Dr. Arnulfo Arias Madrid

\section{REVISIÓN BIBLIOGRÁFICA}

El síndrome de Mirizzi se describe como una complicación de una colelitiasis, donde los litos se impactan en la bolsa de Hartmann o en el conducto cístico y comprimen el conducto biliar. ${ }^{1}$ 
Epidemiología: Se reporta en 0.7 a $3 \%$ de los pacientes con litiasis vesicular. ${ }^{9}$

Fisiopatología: Este cálculo causa una obstrucción mecánica extrínseca de la vía biliar que con el tiempo produce erosión de la pared vesicular e inflamación, formando fístulas colecistocoledocianas. Además, la continua inflamación del triángulo de Calot puede resultar en una fístula hacia cualquier órgano vecino. ${ }^{9}$

Imagen 6. Fisiopatología del síndrome de Mirizzi

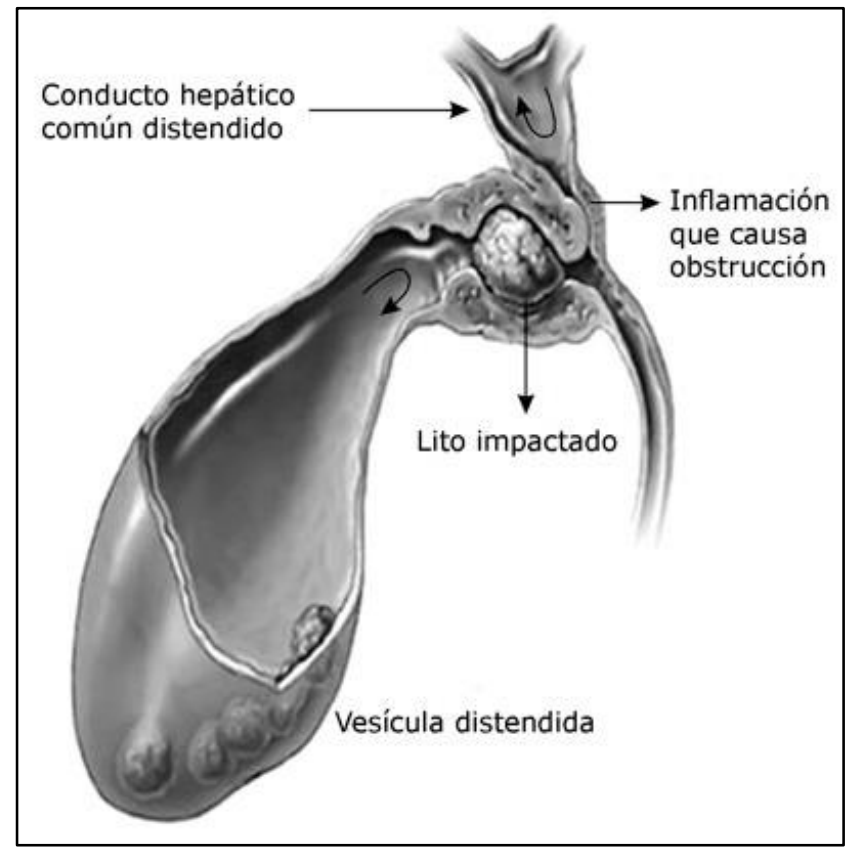

Fuente: Tomado de Galiano Gil JM. El síndrome de Mirizzi, un desafío para el cirujano. Rev. Cub. Cir.

Clasificación: En 1982 Mc Sherry y cols., clasificaron el Síndrome de Mirizzi en 2 tipos; a su vez reclasificados en 1989 por Csendes en cuatro tipos ${ }^{9-}$ 12

I. Compresión extrínseca de la vía biliar por el cálculo impactado.

II. Afectación de $1 / 3$ de la circunferencia de la vía biliar.

III. Afectación de 2/3 de la circunferencia de la vía biliar.

IV. Afectación de toda la circunferencia de la VBP.
Starling subdividió el tipo I en IA y el IB:

Tipo IA si se encuentra un conducto cístico largo que corre paralelo a la vía biliar y se encuentra obstruido por cálculos.

Tipo IB cuando el cístico es corto, encontrándose completamente obliterado por cálculos y no hay fístula.

En 2007, Csendes agrega un V grupo para aquellos casos del I al IV con la presencia de fístula colecistoentérica con o sin íleo biliar. (Ver imagen 7)

Imagen 7. Clasificación del Síndrome de Mirizzi.

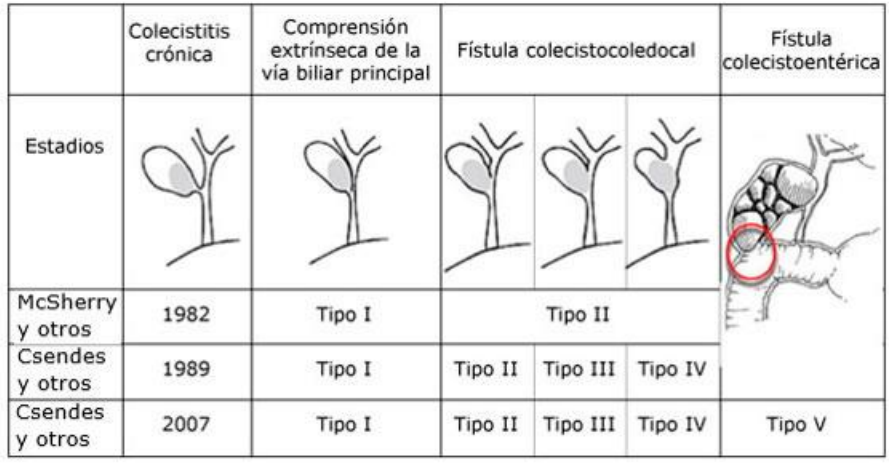

Fuente: Tomado de Galiano Gil JM. El síndrome de Mirizzi, un desafío para el cirujano. Rev. Cub. Cir.

Diagnóstico: El cuadro clínico se puede presentar como una colecistitis aguda, colangitis o pancreatitis aguda; puede acompañarse de hiperbilirrubinemia y leucocitosis. Es indispensable la alta sospecha clínica, la cual se complementa con los estudios de imagen y, en muchos casos, el diagnóstico se realiza de forma intraoperatoria por los hallazgos quirúrgicos encontrados. ${ }^{2}$

Tomografía helicoidal con reconstrucción multiplano: Nos muestra la dilatación de vías biliares proximal a la obstrucción, litiasis gigante en proyección del cuello vesicular. Posee una sensibilidad de $42 \%$ y especificidad de $98.5 \%$ para el diagnóstico de síndrome de Mirizzi. ${ }^{12}$ 
Colangioresonancia: Emerge como tecnología de punta para el diagnóstico de diversas enfermedades. Hace gala de una sensibilidad cercana al $96 \%$ y especificidad de $94 \% .{ }^{9}$

Colangiopancreatografía retrógrada endoscópica (CPRE): La agudeza diagnóstica de la misma oscila entre 55 a $90 \%$ de los casos. Su principal ventaja radica en la posibilidad de ejercer acciones terapéuticas como paliar la obstrucción biliar mediante la colocación de prótesis. ${ }^{9}$

Manejo: La colecistectomía es el gold standard. 2,4 Descompresión temporal mediante la CPRE, usando stents biliares, permite ganar tiempo mientras se mejoran las estrategias quirúrgicas a desarrollar. ${ }^{12}$

Mirizzi tipo I: Colecistectomía laparoscópica, con un plan para convertirse en cirugía abierta si es necesario.

Mirizzi tipo II: La cirugía laparoscópica todavía es posible, sin embargo, plantea un desafío debido a las adherencias densas de la inflamación crónica y la anatomía biliar distorsionada, lo que aumenta el riesgo de lesión biliar.

Tipo III: Derivación bilioentérica. Hepaticoyeyunostomía en $Y$ de Roux.

Tipo IV: Derivación bilioentérica. Hepaticoyeyunostomía en $\mathrm{Y}$ de Roux.

Tipo V: A-Separación de la fístula de la víscera involucrada (duodeno, estómago, colon) y cierre con material absorbible; colecistectomía ya sea total o subtotal de acuerdo a si hay presencia de compresión o fístula colecistobiliar. B- Se recomienda en dos tiempos donde primero se trata la condición asociada (íleo biliar) y en un segundo tiempo se realiza la colecistectomía. ${ }^{2,3,4}$.

\section{CONCLUSIONES}

El síndrome de Mirizzi es una complicación de una colelitiasis de larga data. Es considerada una enfermedad poco común, sin embargo importante; caracterizada por un estrechamiento de la vía biliar por un efecto mecánico e inflamatorio. El diagnóstico preoperatorio del síndrome de Mirizzi es complejo y requiere un alto índice de sospecha para evitar complicaciones serias. A pesar de los avances tecnológicos y en el campo de laboratorio, su diagnóstico es difícil. La falta de sospecha intraoperatoria puede llevar a lesión de la vía biliar con una alta morbilidad y mortalidad. La literatura describe que hasta el momento su manejo ha sido a través de cirugía abierta; sin embargo, cada vez hay más reportes de casos de manejo laparoscópico exitoso en centros de alta complejidad y con experiencia en cirugía laparoscópica.

\section{CONFLICTOS DE INTERESES}

Los autores declaran no tener ningún conflicto de intereses.

\section{AGRADECIMIENTOS}

Agradecemos profundamente a la Dra. Melisa Menéndez, cirujana principal del caso, por su disponibilidad, tiempo y asesoría para el desarrollo del presente caso clínico.

\section{BIBLIOGRAFÍA}

1. Erben Y, Benavente-Chenhalls L, Donohue J, Que $\mathrm{F}$, Kendrick M, Reid-Lombardo K et al. Diagnosis and Treatment of Mirizzi Syndrome: 23-Year Mayo Clinic Experience. Journal of the American College of Surgeons. 2011;213(1):114-119. Disponible en: https://www.ncbi.nlm.nih.gov/pmc/articles/PMC54 49366/

2. Alemi F, Seiser N, Ayloo S. Gallstone Disease. Surgical Clinics of North America. 2019;99(2):231244

Disponible

en:

https://www.researchwithrutgers.com/en/publicati ons/gallstone-disease-cholecystitis-mirizzisyndrome-bouveret-syndrom 
3. Beltrán M. Mirizzi syndrome: History, current knowledge and proposal of a simplified classification. World Journal of Gastroenterology. 2012;18(34):4639-4650. Disponible en: https://www.ncbi.nlm.nih.gov/pmc/articles/PMC34 42202/

4. Asef K, Brack L, Laiño F, Montemarani G, Szmulewicz H, Taboadela F et al. Abordaje Quirúrgico de Síndrome de Mirizzi: Revisión de la Literatura. Pren Méd Argent. 2014;100(4):242-249. Disponible en: https://docplayer.es/63734059-Abordajequirurgico-de-sindrome-de-mirizzi-revision-de-laliteratura.html

5. Cheung F, Mak C, Chu W, Fan N, Lui K. A case of type II Mirizzi syndrome treated by simple endoscopic means. Journal of Surgical Case Reports. 2018;2018(10):1-3. Disponible en: https://academic.oup.com/jscr/article/2018/10/riv2 $\underline{57 / 5123438}$

6. Maple JT, Ben-Menachem T, Anderson MA, et al. The role of endoscopy in the evaluation of suspected choledocholithiasis. Gastrointest Endosc. 2010; 71(1):1-9. doi: 10.1016/j.gie.2009.09.041.

7. Yokoe M, Hata J, Takada T, et al. Tokyo Guidelines 2018: diagnostic criteria and severity grading of acute cholecystitis (with videos). Journal of HepatoBiliary-Pancreatic Sciences. 2018; 25(1):41-54. doi: 10.1002/jhbp.515.

8. Jarnagin WR. Blumgart's Surgery of the Liver, Biliary Tract, and Pancreas. Elsevier; 2016. Disponible en: https://www.elsevier.com/books/blumgartssurgery-of-the-liver-biliary-tract-and-pancreas-2volume-set/jarnagin/978-0-323-34062-5

9. Galiano J. El síndrome de Mirizzi, un desafío para el cirujano. Revista Cubana de Cirugía. 2016;55(2):151-163. Disponible en: http://scielo.sld.cu/pdf/cir/v55n2/cir06216.pdf 10. Clemente G, Tringali A, De Rose A, Panettieri E, Murazio $M$, Nuzzo $G$ et al. Mirizzi Syndrome: Diagnosis and Management of a Challenging Biliary Disease. Canadian Journal of Gastroenterology and Hepatology. 2018;2018:1-6. Disponible en: https://www.researchgate.net/publication/326994
394_Mirizzi_Syndrome_Diagnosis_and_Manageme nt_of_a_Challenging_Biliary_Disease

11. Antoniou S, Antoniou G, Makridis C. Laparoscopic treatment of Mirizzi syndrome: a systematic review. Surgical Endoscopy. 2009;24(1):33-39. Disponible en: https://pubmed.ncbi.nlm.nih.gov/19466486/

12. Milone $M$, Musella $M$, Maietta $P$, Guadioso D, Pisapia A, Coretti G, et al. Acute acalculous cholecystitis determining Mirizzi syndrome: case report and literature review. BMC Surgery. 2014,14:90. Disponible en: https://www.researchgate.net/publication/268451 173 Acute acalculous cholecystitis determining Mirizzi syndrome Case report and literature revi ew 